\title{
LOS ESTUDIOS DE CALIDAD DE VIDA: \\ ALTERNATIVA DE EDUCACIÓN BASADA \\ EN LA INVESTIGACIÓN
}

\author{
Margie N. Jessup C*. y Rosalba Pulido de Castellanos
}

\begin{abstract}
This paper presents some reflections based on both the educational practice and the research experience. Developed by a research group, that seeks to generate conceptual discussion and new educational alternatives focused on life quality studies, it pretends to go further in the analysis to prospect integral formation. It is expected that this approach would lead to adequate: curricula, of projects teaching improvement programs and community participation on the school and plans and programs that take would take into account the life quality conditions of the community to generate formative integral processes and a participate solution to a problems.

It presents a conceptual evolution about life quality as well as concepts of life level, wellbeing and the well-being state. Definitions of life quality and human needs are presented which are of critical importance for the research projects, to determinate contextual conditions of the community as a base to educational action research and educational practices in the formal and non formal fields. An application possibility is shown.
\end{abstract}

\section{PALABRAS CLAVES}

Calidad de vida, desarrollo humano, necesidades humanas, ambiente, investigación, educación.

\section{APROXIMACIONES CONCEPTUALES}

\subsection{Evolución del concepto calidad de vida}

En la literatura es posible hallar reportes que surgen desde la segunda mitad del siglo XIX, basados en una serie de conceptos asociados al que manejamos actualmente sobre calidad de vida, dentro de ellos se destacan condiciones de vida, nivel de vida, bienestar satisfacción felicidad, estilo de vida y otros, considerados por diversos autores en diferentes épocas: en ellos numerosos aspectos relacionados con calidad de vida han sido objeto de investigación y por consiguiente, sus indicadores directos e indirectos forman parte del debate sobre el tema.

\footnotetext{
*Profesora asociada de T.C. Departamento de Biología, Universidad Pedagógica Nacional.

** Profesora asistente de T O., Departamento de Biología. Universidad Pedagógica Nacional. Integrantes de la Línea de Investigación estudios en calidad de vida del Departamento de Biología de la Universidad Pedagógica Nacional 
Se presentan algunos de los más relevantes frente a condiciones de vida, nivel de vida $y$ bienestar, sin pretender en modo alguno abarcarlos todos.

\section{1 Nivel de vida}

En la mayoría de los primeros estudios revisados sobre condiciones de vida que datan aproximadamente de 1884, el nivel de vida se asocia con el nivel de consumo, entendido éste como la cantidad de bienes y servicios utilizados por una población, considerándose sólo aspectos cuantificables y haciendo referencia al grado de satisfacción de las necesidades.

Publicaciones presentadas casi un siglo más tarde tienen prácticamente la misma tendencia con algunas modificaciones así: a) lo consideran como el nivel de satisfacción de las necesidades de la población, de acuerdo con el flujo de bienes y servicios disfrutados por unidad de tiempo, medibles en su mayoría para presentar cuantitativamente sus resultados mediante índices (Drewnowski y Scott, 1968); b) incluyen aspectos nuevos dentro del mismo como son las posesiones culturales de la población estudiada en su contexto, las necesidades "básicas" (Sociedad de Sociología Rural 1956) la estructura demográfica y el ambiente físico y antrópico que viabilicen su satisfacción (Knox. 1974). Es importante destacar que de acuerdo con los planteamientos de este último, tanto la ubicación del estudio como la escala de análisis utilizada inciden en el uso del concepto.

Todos ellos reconocen una estrecha relación entre los aspectos cuantificables y cualificables del nivel de vida y que la medición de los primeros posibilita de alguna manera cierta aproximación a los segundos. Adicionalmente de los estudios referenciados se puede deducir, que al elevar el nivel de vida de una población se dan transformaciones positivas en su estado de bienestar, de ahí la importancia de conocer qué se considera como bienestar y estado de bienestar.

\subsubsection{Bienestar y Estado de Bienestar}

Existen diversos tipos de definición de bienestar. Entre ellos: a) el grado en el cual la totalidad de las necesidades humanas se ve satisfecha en relación con el ambiente propio del grupo considerado (Grupo Sueco-Danés, 1976), que depende del acceso a los bienes y servicios disponibles para una persona o grupo y de su interacción con otras personas, b) un término genérico en el cual se solapan componentes tales como nivel de vida, calidad de vida, satisfacción social, bienestar social y condición de vida (Coates. Johnston y Knox, 1977); c) un sinónimo de desarrollo, pues según ellos éste se refiere al "estar bien de todos y cada uno de los integrantes de una sociedad", grupo poblacional o comunidad. Por consiguiente el ser humano debe considerarse como la razón de ser del desarrollo de las naciones, regiones o áreas geopolíticas, involucrando las diferentes dimensiones de la persona y su entorno. Así las cosas, el logro del desarrollo implica la plena satisfacción de las necesidades para el conjunto de la población en referencia (Banguero, 1982)

No obstante, resultaría inadecuado establecer la connotación de satisfacción de todas las necesidades humanas debido a que ello resulta impracticable en las sociedades actuales, o la satisfacción plena de necesidades, pues dicha satisfacción constituye una tendencia con diferentes grados de ogro, por otra parte, la sinonimia entre desarrollo y bienestar no seria rigurosa pues el primero involucra el bienestar ciudadano. Sin embargo es necesario resaltar que entre desarrollo y bienestar existe relación directa que además 
es biunívoca, pues el auge del bienestar de un individuo o grupo humano contribuye a elevar su calidad de vida, potenciando así su desarrollo y viceversa

El estado de bienestar es considerado por algunos autores como el conjunto de factores que determinan el bienestar ce una determinada población, no puede medirse por unidad de tiempo pues hace referencia a condiciones propias de ella tales como nutrición, educación, salud, empleo y otras. Esto no niega la posibilidad de analizarlos en el tiempo (Drewnowski y Scott, 1968).

Para Dewnowski y Scott (1974) tanto el nivel de vida como el estado de bienestar deben medirse a través de índices independientes y representados en igual forma, pues al haber un incremento en el flujo de bienes y servicios -nivel de vida- de una población, paulatinamente se generan cambios positivos en sus condiciones -estado de bienestar-. Esta distinción permite diferenciar entre bienes y medios de vida que puede poseer una persona o grupo humano, y las condiciones inherentes a dinámica individual y social, en lo relacionado con los aspectos biológicos, sociales, económicos, ambientales y culturales que tienen que ver con su propia naturaleza.

También se encuentran investigadores que agregan en un solo índice flujo de bienes y servicios y existencia de bienestar, considerando que a pesar de haber una estrecha relación entre los aspectos cualificables y cuantificables del nivel de vida, algunos de ellos no son susceptibles de aproximación cuantitativa, cual es el caso de la realización personal y social o la tranquilidad (Knox, 1974).

Revisados sucintamente estos aspectos fundamentales, cabe la pregunta acerca de qué es la calidad de vida.

\subsection{Calidad de vida}

Ya en 1974, Drewnowski asume que la calidad de vida de la población se halla en dependencia tanto del nivel de vida como del estado de bienestar. Para el grupo SuecoDanés por su parte (1976), este concepto consta de dos elementos fundamentales, los cuales se complementan entre si: el bienestar y la satisfacción de las necesidades.

A su vez, Mallman y coautores (1978) consideran la calidad de vida individual como el producto de los estados de salud -entendida como el bienestar físico, mental y social de la población- y satisfacción -asumida como el estado que se precisa al confrontar las aspiraciones con los logros de la persona.

A esta altura se podría inferir de manera global, que los diversos autores conciben directa o indirectamente la calidad de vida como el grado de satisfacción de las necesidades a niveles individual y/o colectivo, satisfacción que a su vez depende tanto de la disponibilidad y accesibilidad de recursos, como del sistema de valores y normas sociales que forman parte de la cultura, la cual a su vez constituye uno de los elementos propios del ambiente humano.

En relación con esto último, resulta de importancia retomar los planteamientos tanto de Coates, Johnston y Knox (1977) acerca de que las diversas condiciones de vida en diferentes sociedades se originan en las estructuras propias de cada una de ellas, como los de Díaz (1985) sobre la necesidad de tener en cuenta las dimensiones económica, política, social y espacial al evaluar aspectos relacionados con las desigualdades sociales, a fin de poder determinar las condiciones de vida humana en un contexto de cambio 
social permanente, en ambos casos subyace la concepción del ambiente como factor incidente en la calidad de vida humana.

Además de lo presentado, Duvigneaud (1984), caracteriza la calidad de vida por la integración de tres conceptos básicos que son: nivel de vida, condiciones de vida y medio de vida. En el planteamiento de este autor, el nivel de vida hace referencia al nivel de ingresos y la acumulación de riqueza, es decir, a la conquista de bienes materiales cada día más abundantes y perfeccionados. Las condiciones de vida están definidas por las características del contexto dentro del cual se desarrollan las actividades humanas: acceso a los servicios públicos, medio de trabajo, medio social, etc.; es decir, se trata de la expresión del contexto social. El medio de vida se define de acuerdo con la calidad del entorno humano en lo físico; esto incluye entre otras cosas: calidad del agua, calidad del aire, disponibilidad de zonas de recreación, etc.

Como puede observarse, esta definición incluye tres aspectos básicos importantes: los económicos, que se incluyen en el nivel de vida (se tiene en cuenta solamente los bienes que se posean); los sociales, que se desarrollan en las condiciones de vida y consideran al individuo en su comunidad. Por último los ambientales, que incluyen el ambiente en el medio de vida; mediante éste se indica la calidad del mismo, en el cual una comunidad se desarrolla. Estos tres componentes se deben enmarcar dentro de un sistema de valores propio de la comunidad a estudiar, sobresaliendo las necesidades y aspiraciones propias de ella (Alfonso, 1988).

El grupo de investigadores de la Fundación Centro de alternativas de Desarrollo (CEPAUR) del cual hace parte M. Max-Neef (1986) indica que la calidad de vida puede constituirse en uno de los mejores indicadores de desarrollo humano, referido no a los objetos sino a las personas, al plantear que el mejor desarrollo es aquel que permite elevar más su calidad de vida estos planteamientos son cercanos a los de Banguero

Cualquiera que sea la definición o caracterización dada, el concepto en si sirve como unidad de evaluación del cambo experimentado por una población; debe permitir tanto la descripción de las modificaciones del sistema como la evaluación ce los mismos en la evolución del sistema propiamente dicho (De la Barra y Rodríguez. 1982) Sin embargo la decisión que se adopte sobre el concepto delimitara su aplicabilidad, determinando el alcance de lo descrito o evaluado, bien sea en una perspectiva limitada o globalizante.

Para el equipo de investigación de esta línea, la calidad de vida de un individuo o grupo humano es su estado de bienestar evaluado a través del grado de satisfacción de sus necesidades en relación con un óptimo de tal satisfacción, en interdependencia con su ambiente (Jessup. M , Pulido de Castellanos, R. 1996). En este sentido, es necesario indicar que para cada persona existe un óptimo, el cual puede constituirse en referente del grado de satisfacción de las necesidades de los grupos humanos que forman parte del ambiente, el cual naturalmente incide en la concepción de óptimo.

Debido a la gran atención que se concede a los aspectos ambientales y la educación ambiental en diferentes ámbitos nacionales e internacionales, la definición de calidad de vida propuesta, al incorporar la interacción del hombre en los niveles individual y social con el ambiente, permite relacionarla con la definición propuesta por Victart (1988), quien lo concibe como el espacio donde el hombre interactúa con la naturaleza y expresa en ella una comunicación simbólica, es decir trabaja se relaciona en sociedad, organiza instituciones sociales, económicas y políticas. Esta dimensión hace posible involucrar la representación que establece el hombre acerca del ambiente, así como de la cultura 
humana de acuerdo con sus procesos históricos, modificando, transformando y adaptando el medio natural y humano en concordancia con sus referentes tecnológicos y de significado.

Por ello, en relación con la definición de calidad de vida que se asume por parte de este grupo ${ }^{1}$, la consideración del ambiente en los términos anteriores tiene una connotación especial, pues de acuerdo con los planteamientos de Sauvé (1994) "la representación del ambiente que una persona o grupo adopten, necesariamente determina sus comportamientos con respecto a ese ambiente, ya sea que se trate de una acción espontánea o de una conducta deliberada".

En la tipología sobre concepciones del ambiente desarrollada por el grupo de L Sauvé, se presentan entre otras dos tendencias: como medio de vida y como medio comunitario, el grupo fundamenta su trabajo en estos dos tipos, pues las acciones basadas en dichas representaciones permiten y exigen la participación ciudadana en procura de la comprensión, conocimiento, transformación y mejoramiento del medio en su acepción más globalizante, en pro de su propio desarrollo.

Así mismo, esta aproximación conceptual sobre calidad de vida exige una concepción de necesidades humanas. A lo largo del tiempo se las ha tratado de definir, identificar, clasificar, medir y priorizar, hecho que ha llevado a diversas estandarizaciones que a pesar de sus diferencias tienen en común lo relativo del concepto necesidad, así como las variaciones que éste presenta en relación con el tiempo, espacio y contexto social a que se refiere Por ello nos detendremos en su definición.

\subsubsection{Necesidades humanas}

Al igual que los anteriores, este concepto ha tenido diferentes tratamientos y utilización por parte de diversos investigadores e instituciones a través del tiempo. Así Mallman. Maslow (Díaz, 1985), Aldelfer (1972) y otros han presentado diversas clasificaciones de necesidades humanas, estos y muchos otros investigadores, consideran que ellas son infinitas, no clasificables y se modifican con el transcurrir del tiempo.

Uno de los grupos que entra en contraposición con esta es el de CEPAUR el cual la califica de suposición incorrecta, producto de un error conceptual, consistente en no diferenciar los conceptos necesidad y satisfactor. Según ellos, las necesidades del ser humano son múltiples e interdependientes y se deben entender como un sistema en el cual se interrelacionan e interactúan, razón por la cual su satisfacción constituye un proceso caracterizado por fenómenos tales como simultaneidades, complementariedades y compensaciones (Max-Neef y col., 1986).

Es necesario indicar, que para el grupo en mención la satisfacción no debe asumirse como algo acabado sino como una aproximación permanente, denominada realización; de igual manera, cada necesidad puede satisfacerse en los términos ya descritos, a diferentes niveles y con diversas intensidades en relación con a) uno mismo. b) el grupo social y c) el ambiente En este punto resulta de utilidad destacar, que los satisfactores elegidos no dependen única y exclusivamente del individuo y el grupo social inmediato al cual pertenece sino también de las instituciones que forman parte de su ambiente nacional.

\footnotetext{
${ }^{1}$ Grupo de investigación Estudios en Calidad de Vida.
} 
En su propuesta Desarrollo a Escala Humana. Una Opción para el Futuro, el grupo propone una clasificación basada en dos posibles criterios de desagregación según categorías existenciales y según categorías axiológicas. La combinación de las mismas incluye por una parte necesidades del ser, tener, hacer y estar y por la otra, necesidades de subsistencia, protección, afecto, entendimiento, participación ocio creación, identidad y libertad, categorías que se pueden combinar en una matriz. En este contexto, llama la atención la existencia de puntos de coincidencia entre los planteamientos de Maslow, Smith y Kaplan previamente mencionados con los de este grupo, en el sentido de considerar algunas necesidades en términos axiológicos.

Tomando como base la clasificación expuesta, M. Neef (1986) explica cómo por ejemplo alimentación y abrigo no deben ser considerados necesidades sino satisfactores de la necesidad fundamental de subsistencia; de igual manera la educación, el estudio, la investigación y otros, constituyen satisfactores de la necesidad de entendimiento y los sistemas curativos y de salud son satisfactores de la necesidad de protección. Así mismo, indica que no existe una correspondencia biunívoca entre necesidades y satisfactores, pues un satisfactor puede contribuir a realizar simultáneamente varias necesidades y viceversa.

En la Línea de Estudios en Calidad de Vida, las necesidades humanas se conciben como carencias totales o parciales de aquellos aspectos biológicos, psicológicos, culturales, económicos, políticos y sociales, indispensables para el desarrollo integral del ser humano y de la sociedad de la cual hace parte, carencias que a su vez se constituyen en potencialidad de dicho desarrollo (Jessup, M., Pulido de Castellanos, R., 1998). Para el caso de este concepto, es preciso puntualizar que el término carencia" trasciende los limites de lo biológico, para ser considerado con referencia al ser humano en su integralidad.

\section{LOS ESTUDIOS DE CALIDAD DE VIDA COMO ALTERNATIVA DE EDUCACION}

\subsection{Consideraciones preliminares}

Antes de analizar la posibilidad propuesta en el subtitulo, resulta de importancia tener en cuenta lo siguiente

- El planteamiento de Andrews (1974) acerca de un acuerdo universal, según el cual un estado moderno posee como una de sus metas legitimas promover el bienestar de sus ciudadanos. Es necesario que de manera global se reconozca, que ésta debe ser la tendencia y cómo se está dejando de lado en sociedades como la nuestra y de países de la región, aún en lo declarativo, lo cual hace de ellos estados atrasados e inequitativos Para el logro del bienestar social se requiere una formación integral.

- Díaz (1985) menciona que dichos estudios, en particular sobre condiciones de vida, surgen como una necesidad de contrarrestar la tendencia de las sociedades occidentales con diferente grado de desarrollo, de dar mayor importancia al crecimiento económico que al bienestar social de los ciudadanos. Aparentemente, algunos estados en la actualidad comienzan a valorar el desarrollo humano como parte esencial del desarrollo nacional, no obstante, un análisis más detallado permite evidenciar que no pasan de ser declaraciones formales y acuerdos multilaterales vacíos, pues en la práctica son cada vez mayores las desigualdades internas y entre países. 
Por ello se perfila cada vez más claramente, la exigencia de formar a nuestros ciudadanos en un espíritu autónomo y crítico, tendiente al desarrollo de sus potencialidades, que les permita evidenciar problemas reales en cuya solución participen desde su ámbito de competencia.

- Las consideraciones de Coates, Johnston y Knox (1977) acerca de que las diversas condiciones de vida en diferentes sociedades se originan en las estructuras propias de cada una de ellas y por consiguiente, muchas de las causas, manifestaciones y consecuencias de las desigualdades son esencialmente estructurales. El evidenciar de manera global dichas desigualdades es posible, si se proporciona a los ciudadanos una sólida formación, que les permita contribuir al desarrollo autónomo del país.

Teniendo en cuenta lo anterior, se puede comprender que la educación concebida como un complejo integral, supone la formación integral de las personas en diferentes ámbitos tales como: intelectual, político e ideológico, ético y moral, estético, lúdico y recreativo, físico, lingüístico y profesional (docente, técnico, médico, etc.). Una educación con estas características, propende por el desarrollo del ser humano y de su sociedad. Sin embargo, si ella está descontextualizada de la realidad del País, resulta carente de sentido para los ciudadanos. Por ello es fundamental conocer las condiciones, problemas y requerimientos de las poblaciones sujeto de educación, esto se logra gracias a su estudio mediante procesos de investigación en todas las etapas (exploración, intervención, evaluación). Por consiguiente, una educación de calidad debe ser de corte investigativo.

\subsection{Formación integral}

Contribuir a la formación integral significa que se requiere viabilizar el desarrollo de las potencialidades y competencias que permitan asumir la propia vida y contribuir adecuadamente al desarrollo social, tanto en los planos familiar como en el comunitario, local y nacional. Esto implica tener en cuenta lo relativo a la enseñanza y al aprendizaje de los campos disciplinares y saberes, como también las interacciones entre los referentes normativos institucionales, las prácticas y comportamientos cotidianos de los miembros de la comunidad educativa (directivos. docentes, alumnos, padres y administradores), en los diferentes ámbitos institucionales.

También hace parte de este proceso la asunción de: a) la "autodeterminación y capacidad crítica y la dignidad de la persona, para aprender a ordenar la vida propia con responsabilidad y libertad" (Gómez, M. 1990) y b) la generación de propuestas de solución a los problemas. Para lograrlo es preciso considerar no sólo la dimensión racional de la persona, sino también su afectividad, sensibilidad, valores, concepción de vida y de sociedad, que determinan y forman parte de su acción.

De una u otra manera la escuela es el centro de desarrollo de conocimientos y experiencias que contribuyen a forjar la personalidad y los rasgos de carácter infantil y juvenil, complementando y/o diferenciando los marcos de referencia propios de sus núcleos familiar barrial. Al respecto cabe mencionar el planteamiento de Torres y Caballero (1990) según el cual en las instituciones educativas al igual que en cualquier otra organización, las personas se relacionan con su realidad a través del trabajo, del lenguaje y de la interacción social. 
Estos autores desarrollan su argumentación con base en las siguientes tesis:

En la escuela, se centra la atención en unos objetivos y una finalidad propia que debe ser:

- Enseñar a desarrollar el pensamiento.

- Enseñar a desarrollar los sentimientos.

- Enseñar a desarrollar la sensibilidad.

En estos tres aspectos debe centrarse el trabajo en una institución educativa. Para realizarlo utiliza el lenguaje en sentido amplio: el lenguaje verbal, el escrito, el fáctico, el simbólico, etc., hasta el lenguaje del silencio...

El trabajo que desempeña la institución educativa es fundamentalmente de interacción social, interacción que se logra a través del lenguaje y en torno a los diferentes tipos de saberes que posee una sociedad." (La negrilla es de los autores).

Esto indica, que los procesos de trabajo no son independientes de la organización institucional y viceversa; así mismo, los procesos sociales se relacionan con la cimentación de tradiciones y cultura.

\subsection{Contexto institucional}

Retomando lo expuesto, es posible considerar que en la institución educativa se generan y afianzan comportamientos, conocimientos, valores, ideologías y en general códigos de acción considerados normales, a través de las interacciones enmarcadas en intereses particulares de los diferentes grupos que constituyen la comunidad. Esto determina una cultura institucional, que influye en la manera como las personas se piensan a si mismas y a los demás y asumen sus propias potencialidades y dificultades.

Aunque la complejidad de la formación ciudadana no es exclusiva de los centros educativos, es claro que en ellos se generan procesos sistemáticos de enseñanzaaprendizaje, organizados de acuerdo con los fines señalados para la educación formal, consecuentemente con la concepción de sociedad y de nación que se quiere construir.

En consecuencia, la confluencia de la cultura institucional y los fines de la educación contribuye a generar opciones de vida y realización de ideales; en otras palabras, allí se viabiliza la capacidad de soñar y "comprometerse en la preparación y ejecución de un proyecto de vida a la medida de las propias ilusiones" (Duque, 1997) en consonancia con el proyecto compartido de nación y sociedad.

Esta afirmación significa que si bien la institución educativa no es la única que propicia la construcción de futuro a nivel individual y colectivo, en ella es posible y pertinente canalizar intereses, compartir inquietudes y pensar la utopía individual al enfrentar la realidad y la cotidianidad del propio vivir, frente a la dinámica propia de los espacios escolares.

Esto es factible en una interacción permanente, por caminos reflexivos y afectivos, involucrando las capacidades de escuchar al otro, de disentir, de actuar y de responder por las acciones. 
Acoger los anteriores planteamientos tiene diferentes consecuencias entre las cuales se encuentra asumir la trascendencia de la escuela como centro de socialización de conocimientos y de formación ciudadana, como organización en la cual coexisten diferentes grupos y como espacio generador de conocimientos pedagógicos y didácticos.

Una de las funciones de la escuela es ser centro de socialización de conocimientos y de formación de ciudadanos, esta función se organiza con base en la corriente de pensamiento (humanismo, empirismo, teoría crítica u otra) que orienta el deber ser y la acción institucional. Dentro de ellas pueden mencionarse entre otras. Según la corriente filosófica prevaleciente, se establecen los fines y metas para el proceso educativo. En todo caso, se reconoce el papel de la educación como: a) acción socializadora y por tanto reproductora de la ideología y patrones de interés de las clases dominantes; b) acción pedagógica y didáctica mediada por intereses de la sociedad, que le son planteados mediante leyes y normas generales; c) la interacción profesor-alumno como proceso en el cual se presentan relaciones de poder y autoridad sobre el saber, que inciden en los resultados del proceso educativo.

Como organización en la cual coexisten grupos, en la escuela se pueden cimentar entre otros los siguientes: grupos de maestros que procuran organizar currículum adecuados, constituirse como comunidad académica, unirse en torno a asuntos gremiales y laborales, de alumnos en lo relacionado con la participación y desarrollo de actividades extracurriculares, de bienestar y participación en el gobierno escolar: de familiares y representantes de organizaciones gubernamentales y cívicas relacionados con el centro educativo, que trabajan en pro del desarrollo institucional en cuanto a su infraestructura y servicios adecuados a las expectativas del colectivo

Igualmente la escuela tiene la posibilidad de trascender sus límites geográficos, mediante acciones la realización de que contribuyan a generar conciencia sobre la problemática local y la posibilidad de enfrentarla mancomunadamente, con el objeto de gestar soluciones desde el colectivo y/o con el apoyo de instituciones encargadas de atender determinados campos en la sociedad. Cualquiera que sea el tipo de agrupación considerada en la institución, las interacciones que se originan involucran intereses, problemas y situaciones particulares, de sus miembros, Como grupo es posible que se tienda a influir sobre la toma de decisiones.

Adicionalmente, la escuela es un espacio generador de conocimientos pedagógicos y didácticos. Como ya se enunció, en ella se realiza el proceso educativo de manera sistemática; por eso constituye el espacio natural de desarrollo de conocimientos sobre enseñanza y aprendizaje, la relación maestro alumno, los procedimientos, estrategias y dinámicas recomendables para el trabajo con determinados grupos escolares y temáticas particulares.

El reto está en que no basta con organizar los planes y programas de una manera sistemática, se requiere ante todo el desarrollo de procesos de seguimiento reflexión, debate entre los integrantes de la comunidad, sistematización y divulgación de las experiencias educativas ...en fin, se hace necesario incorporar en el quehacer educativo la interrelación teoría-práctica como acción consubstancial al trabajo docente, que no puede ser relegada ante las urgencias de atender clases y otras actividades escolares, en detrimento del desarrollo de conocimientos pedagógicos y didácticos 


\section{ESTUDIOS EN CALIDAD DE VIDA Y EDUCACION}

\subsection{Fundamentación}

La investigación en la perspectiva que se aborda en este trabajo no se centra en el desarrollo de conocimientos en una disciplina particular, sino en una práctica sistemática, asumida como resultado de la interrelación permanente entre la reflexión y la acción crítica. Su objetivo consiste en cimentar procesos de formación integral de los educandos, teniendo en cuenta las características biológicas, sociales, culturales y políticas que determinan sus condiciones de vida. Por tanto este tipo de formación involucra el desarrollo de habilidades para pensar y actuar críticamente, con sentido ético, afectividad y sensibilidad, tanto en lo individual como en lo social.

El pensamiento y la acción crítica están en estrecha relación con la libertad y la responsabilidad, vinculadas al desarrollo de la sociedad como resultado del mejoramiento de la calidad de vida de todos. En esta perspectiva tienen relevancia tanto las potencialidades individuales como la realización personal puestas al servicio del bien común. La responsabilidad del desarrollo de las mismas es competencia -entre otros- de la educación formal.

Por consiguiente, se propone involucrar en el proyecto educativo institucional desarrollos investigativos en torno a aspectos que permitan caracterizar grupos o comunidades especificas, estableciendo las problemáticas susceptibles de abordaje educativo tanto en los currículos formales, como en los procesos de cualificación a padres y a otros integrantes de la escuela. De ello se deduce que la investigación no es una etapa inicial; más bien se constituye en un eje orientador y regulador de las acciones planeadas y de la prospectiva institucional en sus diferentes áreas de atención: académica (currículo, educación no formal, actividades extracurriculares), bienestar, servicios de apoyo, administración y organización escolar, entre otros.

Para potenciar la acción y enriquecer la experiencia formativa de los integrantes de una comunidad, es necesario tener en cuenta sus características y las de los grupos poblacionales con los cuales se realiza el proceso; ello permitirá considerar la realidad particular que enfrentan los estudiantes y los docentes, la cual puede constituirse en información valiosa para planear jas acciones educativas, permitiendo asumir la cotidianidad extramural como aspecto relevante en el trabajo.

Esto es apremiante si se tiene en cuenta que aún en una misma área geográfica, rural o urbana, existen contrastes entre las condiciones de vida y los satisfactores de necesidades en los diferentes grupos humanos que en ella habitan; en ocasiones, entre ellos es posible hallar diferencias extremas, aún en sus expectativas de futuro y sus posibilidades de logro. Entre los aspectos contrastantes se encuentran los relacionados con condiciones de vida (ej.: patrones culturales y valorativos) en una relación multidimensional, siendo ellos mismos causas y consecuencias de condiciones sociales, económicas, afectivas, cívicas, educativas y relacionales.

La situación expuesta anteriormente es ni más ni menos el reflejo de las desigualdades sociales, en las cuales se alimenta el desarrollo de violencia, intolerancia y conflicto, aunado con el desarraigo y pérdida del sentido de lo colectivo y del bien común frente al individualismo. 
La afirmación sobre la pertinencia de tener en cuenta las particularidades de los sujetos que interactúan en el proceso educativo, se evidencia al tener en cuenta que en el propósito de propender por la formación integral de la persona, le "cabe a los profesores asumir la responsabilidad del conocimiento que organizan, producen, median, recrean y trasladan a la práctica de la cultura". (Giroux, 1997).

Este mismo autor sostiene que es necesario "volver a estructurar el currículum para definir de nuevo lo cotidiano como un recurso importante para vincular las escuelas con las tradiciones, comunidades e historias que proporcionan a los estudiantes un sentido de voz y relación con otros".

Los estudios relacionados con la calidad de vida aportan conocimiento sobre el contexto y marcos de referencia particulares de los estudiantes y demás integrantes de las comunidades educativas, de manera que pueden contribuir a fundamentar los planes y programas institucionales, así como el desarrollo de currículum adecuados a los grupos a quienes van dirigidos. Esto no significa minimización del reto de contribuir a la consolidación de utopías (esperanzas y empeño para lograr consolidar proyectos de vida) y trabajo solidario, como medios para rescatar la diferencia en una perspectiva de construcción de ciudadanía plena, es decir, del logro de un desarrollo integral de las personas en el horizonte de construcción de procesos críticos, tendientes al desarrollo de la democracia.

\subsection{Aplicabilidad}

Partiendo de una actitud y una práctica investigativa, tendientes a identificar las características del contexto intra y extraescolar a fin de organizar y llevar a cabo procesos educativos de calidad, es posible determinar problemas que inciden en la calidad de vida de los integrantes de dicha comunidad, susceptibles de abordaje educativo.

Son varios los factores que favorecerían la generación de alternativas con sentido para todos los padres de familia y las organizaciones que forman parte del área de influencia de la institución educativa anhelan solucionar problemas sentidos, el maestro desea motivar a sus estudiantes y que ellos logren aprendizajes relevantes, a través de la realización de esos procesos. El estudiante desea que su trabajo sea útil; la sociedad espera (a veces desproporcionadamente) soluciones desde la escuela y ésta desearía generar una educación liberadora y creativa. Por ello no resulta difícil organizar esos diferentes estamentos en torno a la búsqueda de sentidos educativos y al desarrollo de procesos de formación adecuados; lo difícil consiste en poner en práctica un proceso armónico que permita su organización.

En el caso de esta línea, a partir de la fundamentación teórica expuesta y por consiguiente de la categorización de necesidades y satisfactores planteada por el grupo CEPAUR, el trabajo se ha podido organizar alrededor de satisfactores de necesidades tanto en el plano axiológico como ontológico: educar hacia la construcción de identidad y de dignidad humana, de proyectos de vida, de comunidad; hacía el reconocimiento del otro y la minimización de la violencia; hacia la autonomía; educar para elevar la autoestima; educar para el logro de salud (por ejemplo, educar para alimentarse adecuadamente con los recursos a los cuales se tiene acceso), etc... Se trabaja con todos los miembros de la comunidad educativa, pero fundamentalmente con escolares, padres y docentes, en un ejercicio permanente de reflexión y acción. 
El trabajo de apoyo al desarrollo investigativo, como base de la formación de los licenciados, se ha estructurado en varias direcciones a partir de diferentes áreas: docencia, investigación y proyección institucional.

En la de docencia, a través de:

- La ejecución de procesos de práctica pedagógica enmarcados en un proyecto, buscando reflexionar críticamente sobre la acción docente, analizar cada uno de los elementos que componen la realidad escolar y diseñando alternativas didácticas a problemas concretos detectados en la acción educativa.

- La realización de trabajos de semestre, complementarios y de integración de la formación básica en los diferentes componentes académicos del programa de Licenciatura en Biología.

- La ejecución de trabajos de grado, con acercamiento investigativo.

- La formación a través de cursos electivos generales o de profundización en un tema particular.

En la de investigación, mediante el desarrollo de proyectos del equipo de investigación con participación de estudiantes de la licenciatura en Biología.

En la de proyección institucional, a través de:

- La puesta en marcha de procesos conjuntos con otras unidades académicas de la Universidad y otras instituciones.

- La participación en eventos de carácter nacional e internacional.

- Publicaciones.

- Participación en cursos nacionales e internacionales relacionados con educación ambiental y en población.

- Contribución a la generación y desarrollo de planes y programas de trabajo, investigación y formación en el nivel de postgrado

- Participación en procesos de formación continuada de docentes en ejercicio.

- Otros.

\section{BIBLIOGRAFIA}

ALDERFER, C. P. 1972. Existence, Relatednes and Growth: Human Needs in Organizational Settings. New York. Free press. En: SCHEIN, E. 1982. Psicología de la Organización. México. Prentice Hall Hispanoamericana. 252 p. 
ALFONSO, N. 1988. Análisis y caracterización de la calidad de vida en la cabecera del municipio de Tibaná. Tesis de Grado. Universidad Javeriana. Bogotá.

ANDREWS, F. 1974. Social Indicators and Perceived Life Qualíty. En: DIAZ, K. 1985. Los estudios geográficos sobre la calidad de vida en Venezuela. Rey. Geográfica 102, pp. 55-72.

BANGUERO, H. 1982. Colombia 2000: Estrategias de desarrollo para satisfacer las necesidades humanas esenciales en Colombia. Universidad de los Andes CEDE $N^{0} 4$. Editor Harold Enrique Banguero Lozano. Bogotá, Uniandes 326 p Serie: Colección Debates.

COATES, B.; JONHSTON, R. and KNOX, P. 1977. Geography and Inequality. Oxford University Press. England. En: DIAZ, K. 1985. Los estudios geográficos sobre la calidad de vida en Venezuela. Rev. Geográfica 102, pp. 55-72.

DANISH-SWEDISH GROUP FOR UNITED NATIONS. 1976. Economic Comission for Europe. Quality of Life in Urban Satlements Comitee for Housing Building and Regional Research Danish Swedish Version. January. En: DIAZ, K. 1985. Los estudios geográficos sobre la calidad de vida en Venezuela. Rev. Geográfica 102, pp. 55-72.

De la BARRA y RODRÍGUEZ. 1982. En: ALFONSO, N. 1988. Análisis y caracterización de la calidad de vida en la cabecera del municipio de Tibaná. Tesis de Grado. Universidad Javeriana. Bogotá.

DÍAZ, K. 1985. Los estudios geográficos sobre la calidad de Vida en Venezuela. Rey. Geográfica 102, pp. 55-72.

DREWNOSWSKY, J. 1974. On Measuring and Planning the Quality of Life. Publication of the institute for Social Studies. Paperback Series y. XI. Mouton. En: DIAZ. K 1985 Los estudios geográficos sobre la calidad de vida en Venezuela. Rev Geográfica 102, PP. 55-72.

DREWNOWSKY J . SCOTT. W. 1968. The Level of Living index. U N Research institute for Social Development, Report N. 4 Geneve. Sept En DÍAZ, K 1985. Los estudios geográficos sobre la calidad de vida en Venezuela. Rey. Geográfica 102. pp 55-72.

DUQUE. O. 1997. invitación a Disoñar. En: Disoñadores del futuro. Para cambiar el rumbo. Asociación para el Desarrollo Campesino. Fundación Colombia Multicolor. Pasto. Colombia.

DUVIGNEAUD. P 1984 La Sinthese Ecologique. $3^{\text {er }}$ tirage Dom Editours, Paris. En: ALFONSO, N 1988 Análisis y caracterización de la calidad de vida en la cabecera del municipio de Tibaná. Tesis de Grado Universidad Javeriana. Bogotá.

GIROUX. H. 1997 Cruzando limites. Trabajadores culturales y políticas educativas. Barcelona Paidós.

GÓMEZ. M Teoría crítica y educación. Argumentos. 24/25 26/27 Bogotá, Ediciones ECOE pp. 105-150. 
JESSUP, M.; PULIDO DE CASTELLANOS, R. 1996. Estudios en calidad de vida Documento de trabajo Departamento de Biología. Universidad Pedagógica Nacional. Santafé de Bogotá.

KNOX. P 1975 Social Well-being: A spatial Perspective. Oxford University Press. England. En DÍAZ, K. 1985. Los estudios geográficos sobre la calidad de vida en Venezuela. Rev. Geográfica 102, pp.55-72.

MALLMAN, O.: MAX-NEEF, M. and NUDLER, O. 1978. Notes on Meanful and Practical Measures of Health, satisfaction and Quality of Life. Fundación Bariloche. Argentina. En: DIAZ, K. 1985. Los estudios geográficos sobre la calidad de vida en Venezuela. Rev. Geográfica 102, pp. 55-72.

MASLOW, A. 1954. Motivación y personalidad. New York. Harper.

MAX-NEEF, M. y col. 1986. Desarrollo a escala humana. Centro de Alternativas de Desarrollo. CEPAUR, Fundación Dag Hammarskjóld Santiago. Nordan.

RURAL SOCIOLOGICAL SOCIETY 1956. Sociological Research into Rural Levels and Standards of Living. Rural Sociology N. 21 En: DIAZ, K. 1985. Los estudios geográficos sobre la calidad de vida en Venezuela. Rev. Geográfica 102, pp 55-72

SAUVE, L. 1994. Exploración de conceptos y prácticas en la educación relativa al ambiente. La dimensión ambiental y la escuela. II Seminario Internacional. Memorias. Serie Documentos Especiales MEN y FES. Santafé de Bogotá.

SIERRA BARRENECHE, E. 1990. En: DNP. Memorias del Seminario Internacional: Gestión de Calidad y Productividad como Estrategia para el Desarrollo. Cartagena, junio 13-15.

TORRES, M.: CABALLERO, J. L. Conocer para transformar. Una experiencia de investigación-acción participativa. Fundación para la investigación, la Educación y el Desarrollo. Blanecolor Ltda. Manizales. 1990.

VIDART, D. 1988. La educación en materia ambiental: un modelo sistemático para reciclaje de docentes. Educación Hoy. Perspectivas Latinoamericanas. 